\title{
Using Area Mean Value Theorem to Solve Some Double Integrals
}

\author{
Chii-Huei $\mathbf{Y u}^{1, *}$, Shinn-Der Sheu ${ }^{2}$ \\ ${ }^{1}$ Department of Management and Information, Nan Jeon University of Science and Technology, Tainan City, Taiwan \\ ${ }^{2}$ Department of Information Technology, Nan Jeon University of Science and Technology, Tainan City, Taiwan \\ *Corresponding author: chiihuei@nju.edu.tw
}

Received May 11, 2014; Revised June 18, 2014; Accepted June 26, 2014

\begin{abstract}
The present paper studies six types of double integrals and uses Maple for verification. These double integrals can be solved using area mean value theorem. On the other hand, some examples are used to demonstrate the calculations.
\end{abstract}

Keywords: double integrals, area mean value theorem, Maple

Cite This Article: Chii-Huei Yu, and Shinn-Der Sheu, "Using Area Mean Value Theorem to Solve Some Double Integrals." Turkish Journal of Analysis and Number Theory, vol. 2, no. 3 (2014): 75-79. doi: 10.12691/tjant-2-3-4.

\section{Introduction}

In calculus and engineering mathematics, there are many methods to solve the integral problems including change of variables method, integration by parts method, partial fractions method, trigonometric substitution method, and so on. In this paper, we study the following six types of double integrals which are not easy to obtain their answers using the methods mentioned above.

$$
\begin{aligned}
& \int_{0}^{2 \pi} \int_{0}^{R} A(r, \theta, s, \phi, n) d r d \theta, \\
& \int_{0}^{2 \pi} \int_{0}^{R} B(r, \theta, s, \phi, n) d r d \theta, \\
& \int_{0}^{2 \pi} \int_{0}^{R} C(r, \theta, s, \phi, n) d r d \theta, \\
& \int_{0}^{2 \pi} \int_{0}^{R} D(r, \theta, s, \phi, n) d r d \theta, \\
& \int_{0}^{2 \pi} \int_{0}^{R} E(r, \theta, s, \phi, n) d r d \theta, \\
& \int_{0}^{2 \pi} \int_{0}^{R} F(r, \theta, s, \phi, n) d r d \theta,
\end{aligned}
$$

where $s, \phi, R$ are real numbers, $R>0$, and $n$ is a positive integer,

$$
\begin{aligned}
& A(r, \theta, s, \phi, n) \\
& =r \exp \left[\sum_{k=0}^{n} \frac{(n)_{k}}{k !} s^{n-k} r^{k} \cos [(n-k) \phi+k \theta]\right] \\
& \times \cos \left[\sum_{k=0}^{n} \frac{(n)_{k}}{k !} s^{n-k} r^{k} \sin [(n-k) \phi+k \theta]\right],
\end{aligned}
$$

$$
\begin{aligned}
& B(r, \theta, s, \phi, n) \\
& =r \exp \left[\sum_{k=0}^{n} \frac{(n)_{k}}{k !} s^{n-k} r^{k} \cos [(n-k) \phi+k \theta]\right] \\
& \times \sin \left[\sum_{k=0}^{n} \frac{(n)_{k}}{k !} s^{n-k} r^{k} \sin [(n-k) \phi+k \theta]\right], \\
& C(r, \theta, s, \phi, n) \\
& =r \sin \left[\sum_{k=0}^{n} \frac{(n)_{k}}{k !} s^{n-k} r^{k} \cos [(n-k) \phi+k \theta]\right] \\
& \times \cosh \left[\sum_{k=0}^{n} \frac{(n)_{k}}{k !} s^{n-k} r^{k} \sin [(n-k) \phi+k \theta]\right], \\
& D(r, \theta, s, \phi, n) \\
& =r \cos \left[\sum_{k=0}^{n} \frac{(n)_{k}}{k !} s^{n-k} r^{k} \cos [(n-k) \phi+k \theta]\right] \\
& \times \sinh \left[\sum_{k=0}^{n} \frac{(n)_{k}}{k !} s^{n-k} r^{k} \sin [(n-k) \phi+k \theta],\right. \\
& E(r, \theta, s, \phi, n) \\
& =r \cos \left[\sum_{k=0}^{n} \frac{(n)_{k}}{k !} s^{n-k} r^{k} \cos [(n-k) \phi+k \theta]\right] \\
& \times \cosh \left[\sum_{k=0}^{n} \frac{(n)_{k}}{k !} s^{n-k} r^{k} \sin [(n-k) \phi+k \theta],\right. \\
&
\end{aligned}
$$

and

$$
\begin{aligned}
& F(r, \theta, s, \phi, n) \\
& =r \sin \left[\sum_{k=0}^{n} \frac{(n)_{k}}{k !} s^{n-k} r^{k} \cos [(n-k) \phi+k \theta]\right] \\
& \times \sinh \left[\sum_{k=0}^{n} \frac{(n)_{k}}{k !} s^{n-k} r^{k} \sin [(n-k) \phi+k \theta]\right]
\end{aligned}
$$


We can obtain the solutions of these double integrals using area mean value theorem; these are the major results of this paper (i.e., Theorems 1-3). Adams et al. [1], Nyblom [2], and Oster [3] provided some techniques to solve the integral problems. $\mathrm{Yu}$ [4-29], $\mathrm{Yu}$ and $\mathrm{B}$. - $\mathrm{H}$. Chen [30], and T. -J. Chen and $\mathrm{Yu}[31,32,33]$ used complex power series method, integration term by term theorem, differentiation with respect to a parameter, Parseval's theorem, and generalized Cauchy integral formula to solve some types of integrals. In this paper, three examples are used to demonstrate the proposed calculations, and the manual calculations are verified using Maple.

\section{Main Results}

Some formulas and theorems used in this paper are introduced below.

\subsection{Euler's Formula}

$e^{i x}=\cos x+i \sin x$, where $i=\sqrt{-1}$, and $x$ is any real number.

\subsection{DeMoivre's Formula}

$(\cos x+i \sin x)^{m}=\cos m x+i \sin m x$, where $m$ is any integer, and $x$ is any real number.

The following Formula 2.3 and Formula 2.4 can be found in [[34], p62]

\section{3.}

$\sin (a+i b)=\sin a \cosh b+i \cos a \sinh b$, where $a, b$ are real numbers.

\section{4.}

$\cos (a+i b)=\cos a \cosh b-i \sin a \sinh b$, where $a, b$ are real numbers.

\subsection{Binomial Theorem:}

$(u+v)^{n}=\sum_{k=0}^{n} \frac{(n)_{k}}{k !} u^{n-k} v^{k}$, where $u, v$ are complex numbers, $n$ is a positive integer, $(n)_{k}=n(n-1) \cdots(n-k+1)$ for positive integers $k$, and $(n)_{0}=1$.

An important theorem used in this study is introduced below, which can be found in [[35], p147].

\subsection{Area Mean Value Theorem}

Suppose that $z, \lambda$ are complex numbers, and $R>0$. If $f(z)$ is analytic in a domain which contains the closed $\operatorname{disc}\{z \in C|| z-\lambda \mid \leq R\}$, then:

$$
\frac{1}{\pi R^{2}} \iint_{|z-\lambda| \leq R} f(z) r d r d \theta=f(\lambda) .
$$

Firstly, we determine the solutions of the double integrals (1) and (2).
Theorem 1 Suppose that $s, \phi, R$ are real numbers, $R>0$, and $n$ is a positive integer. Then the double integrals

$$
\begin{aligned}
& \int_{0}^{2 \pi} \int_{0}^{R} A(r, \theta, s, \phi, n) d r d \theta \\
& =\pi R^{2} \exp \left(s^{n} \cos n \phi\right) \cdot \cos \left(s^{n} \sin n \phi\right),
\end{aligned}
$$

and

$$
\begin{aligned}
& \int_{0}^{2 \pi} \int_{0}^{R} B(r, \theta, s, \phi, n) d r d \theta \\
& =\pi R^{2} \exp \left(s^{n} \cos n \phi\right) \cdot \cos \left(s^{n} \sin n \phi\right),
\end{aligned}
$$

where

$$
\begin{aligned}
& A(r, \theta, s, \phi, n) \\
& =r \exp \left[\sum_{k=0}^{n} \frac{(n)_{k}}{k !} s^{n-k} r^{k} \cos [(n-k) \phi+k \theta]\right] \\
& \times \cos \left[\sum_{k=0}^{n} \frac{(n)_{k}}{k !} s^{n-k} r^{k} \sin [(n-k) \phi+k \theta]\right]
\end{aligned}
$$

and

$$
\begin{aligned}
& B(r, \theta, s, \phi, n) \\
& =r \exp \left[\sum_{k=0}^{n} \frac{(n)_{k}}{k !} s^{n-k} r^{k} \cos [(n-k) \phi+k \theta]\right] \\
& \times \sin \left[\sum_{k=0}^{n} \frac{(n)_{k}}{k !} s^{n-k} r^{k} \sin [(n-k) \phi+k \theta]\right] .
\end{aligned}
$$

Proof Using area mean value theorem for analytic function $f(z)=\exp \left(z^{n}\right)$ yields:

$$
\int_{0}^{2 \pi} \int_{0}^{R} \exp \left[\left(z+r e^{i \theta}\right)^{n}\right] r d r d \theta=\pi R^{2} \exp \left(z^{n}\right) .
$$

Let $z=s e^{i \phi}$, then by Euler's formula, DeMoivre's formula and binomial theorem, we obtain:

$$
\begin{aligned}
& \int_{0}^{2 \pi} \int_{0}^{R} r \exp \left[\sum_{k=0}^{n} \frac{(n)_{k}}{k !}\left(s e^{i \phi}\right)^{n-k}\left(r e^{i \theta}\right)^{k}\right] d r d \theta \\
& =\pi R^{2} \exp \left(s^{n} e^{i n \phi}\right) .
\end{aligned}
$$

Thus,

$$
\begin{aligned}
& \int_{0}^{2 \pi} \int_{0}^{R} r \exp \left[\sum_{k=0}^{n} \frac{(n)_{k}}{k !} s^{n-k} r^{k} e^{i[(n-k) \phi+k \theta]}\right] d r d \theta \\
& =\pi R^{2} \exp \left(s^{n} \cos n \phi+i s^{n} \sin n \phi\right) .
\end{aligned}
$$

Using the equality of real parts of both sides of Eq. (17) yields Eq. (13) holds. Also by the equality of imaginary parts of both sides of Eq. (17), we obtain Eq. (14).

Next, the solutions of the double integrals (3) and (4) can be obtained below.

Theorem 2 If the assumptions are the same as Theorem 1, then the double integrals

$$
\begin{aligned}
& \int_{0}^{2 \pi} \int_{0}^{R} C(r, \theta, s, \phi, n) d r d \theta \\
& =\pi R^{2} \sin \left(s^{n} \cos n \phi\right) \cdot \cosh \left(s^{n} \sin n \phi\right),
\end{aligned}
$$

and 


$$
\begin{aligned}
& \int_{0}^{2 \pi} \int_{0}^{R} D(r, \theta, s, \phi, n) d r d \theta \\
& =\pi R^{2} \cos \left(s^{n} \cos n \phi\right) \cdot \sinh \left(s^{n} \sin n \phi\right),
\end{aligned}
$$

where

$$
\begin{aligned}
& C(r, \theta, s, \phi, n) \\
& =r \sin \left[\sum_{k=0}^{n} \frac{(n)_{k}}{k !} s^{n-k} r^{k} \cos [(n-k) \phi+k \theta]\right] \\
& \times \cosh \left[\sum_{k=0}^{n} \frac{(n)_{k}}{k !} s^{n-k} r^{k} \sin [(n-k) \phi+k \theta]\right],
\end{aligned}
$$

and

$$
\begin{aligned}
& D(r, \theta, s, \phi, n) \\
& =r \cos \left[\sum_{k=0}^{n} \frac{(n)_{k}}{k !} s^{n-k} r^{k} \cos [(n-k) \phi+k \theta]\right] \\
& \times \sinh \left[\sum_{k=0}^{n} \frac{(n)_{k}}{k !} s^{n-k} r^{k} \sin [(n-k) \phi+k \theta]\right] .
\end{aligned}
$$

Proof By area mean value theorem for analytic function $g(z)=\sin \left(z^{n}\right)$, we have:

$$
\int_{0}^{2 \pi} \int_{0}^{R} \sin \left[\left(z+r e^{i \theta}\right)^{n}\right] r d r d \theta=\pi R^{2} \sin \left(z^{n}\right) .
$$

Let $z=s e^{i \phi}$, then:

$$
\begin{aligned}
& \int_{0}^{2 \pi} \int_{0}^{R} r \sin \left[\sum_{k=0}^{n} \frac{(n)_{k}}{k !}\left(s e^{i \phi}\right)^{n-k}\left(r e^{i \theta}\right)^{k}\right] d r d \theta \\
& =\pi R^{2} \sin \left(s^{n} e^{i n \phi}\right) .
\end{aligned}
$$

It follows that:

$$
\begin{aligned}
& \int_{0}^{2 \pi} \int_{0}^{R} r \sin \left[\sum_{k=0}^{n} \frac{(n)_{k}}{k !} s^{n-k} r^{k} e^{i[(n-k) \phi+k \theta]}\right] d r d \theta \\
& =\pi R^{2} \sin \left(s^{n} \cos n \phi+i s^{n} \sin n \phi\right) .
\end{aligned}
$$

By Formula 2.3 and the equality of real parts of both sides of Eq. (22), we obtain Eq. (18). Also using Formula 2.3 and the equality of imaginary parts of both sides of Eq. (22) yields Eq. (19) holds.

Finally, we solve the double integrals (5) and (6).

Theorem 3 If the assumptions are the same as Theorem 1 , then the double integrals

$$
\begin{aligned}
& \int_{0}^{2 \pi} \int_{0}^{R} E(r, \theta, s, \phi, n) d r d \theta \\
& =\pi R^{2} \cos \left(s^{n} \cos n \phi\right) \cdot \cosh \left(s^{n} \sin n \phi\right),
\end{aligned}
$$

and

$$
\begin{aligned}
& \int_{0}^{2 \pi} \int_{0}^{R} F(r, \theta, s, \phi, n) d r d \theta \\
& =\pi R^{2} \sin \left(s^{n} \cos n \phi\right) \cdot \sinh \left(s^{n} \sin n \phi\right),
\end{aligned}
$$

where

$$
\begin{aligned}
& E(r, \theta, s, \phi, n) \\
& =r \cos \left[\sum_{k=0}^{n} \frac{(n)_{k}}{k !} s^{n-k} r^{k} \cos [(n-k) \phi+k \theta]\right] \\
& \times \cosh \left[\sum_{k=0}^{n} \frac{(n)_{k}}{k !} s^{n-k} r^{k} \sin [(n-k) \phi+k \theta]\right],
\end{aligned}
$$

and

$$
\begin{aligned}
& F(r, \theta, s, \phi, n) \\
& =r \sin \left[\sum_{k=0}^{n} \frac{(n)_{k}}{k !} s^{n-k} r^{k} \cos [(n-k) \phi+k \theta]\right] \\
& \times \sinh \left[\sum_{k=0}^{n} \frac{(n)_{k}}{k !} s^{n-k} r^{k} \sin [(n-k) \phi+k \theta]\right] .
\end{aligned}
$$

Proof Using area mean value theorem for analytic function $h(z)=\cos \left(z^{n}\right)$ and Formula 2.4, we can easily obtain the desired results.

\section{Examples}

In the following, for the six types of double integrals in this study, we provide some examples and use Theorems 1-3 to determine their solutions. On the other hand, Maple is used to calculate the approximations of some double integrals and their solutions for verifying our answers.

Example 1 In Eq. (13), let $s=2, \phi=\frac{\pi}{3}, n=1$, and $R=2$, we obtain:

$$
\int_{0}^{2 \pi} \int_{0}^{2} A\left(r, \theta, 2, \frac{\pi}{3}, 1\right) d r d \theta=4 \pi e \cdot \cos (\sqrt{3}),
$$

where

$$
A\left(r, \theta, 2, \frac{\pi}{3}, 1\right)=r \exp (1+r \cos \theta) \cdot \cos (\sqrt{3}+r \sin \theta) .
$$

Next, we use Maple to verify the correctness of Eq. (25) $>$ evalf(Doubleint $\left(\mathrm{r}^{*} \exp (1+\mathrm{r} * \cos (\text { theta }))^{*} \cos \left(\operatorname{sqrt}(3)+\mathrm{r}^{*} \sin \right.\right.$ (theta)),r=0..2, theta $=0 . .2 * \mathrm{Pi}), 18$ );

$$
-5.48444066856117700
$$

$>\operatorname{evalf}\left(4 * \mathrm{Pi}^{*} \exp (1) * \cos (\operatorname{sqrt}(3)), 18\right)$;

\section{$-5.48444066856117688$}

Also using Eq. (14) yields:

$$
\int_{0}^{2 \pi} \int_{0}^{2} B\left(r, \theta, 2, \frac{\pi}{3}, 1\right) d r d \theta=4 \pi e \cdot \sin (\sqrt{3})
$$

where

$$
B\left(r, \theta, 2, \frac{\pi}{3}, 1\right)=r \exp (1+r \cos \theta) \cdot \sin (\sqrt{3}+r \sin \theta) .
$$

$>$ evalf(Doubleint $\left(\mathrm{r}^{*} \exp \left(1+\mathrm{r}^{*} \cos (\right.\right.$ theta $\left.)\right) * \sin \left(\operatorname{sqrt}(3)+\mathrm{r}^{*} \sin \right.$ (theta)),r=0..2, theta $=0 . .2 * \mathrm{Pi}), 18)$;

$$
33.7157808756591908
$$

$>\operatorname{evalf}\left(4 * \mathrm{Pi}^{*} \exp (1) * \sin (\operatorname{sqrt}(3)), 18\right)$;

\subsection{9}

Example 2 In Eq. (18), if $s=1, \phi=\frac{\pi}{2}, n=2$, and $R=3$, then:

$$
\int_{0}^{2 \pi} \int_{0}^{3} C\left(r, \theta, 1, \frac{\pi}{2}, 2\right) d r d \theta=-9 \pi \sin (1)
$$

where 


$$
\begin{aligned}
C\left(r, \theta, 1, \frac{\pi}{2}, 2\right)= & r \sin \left(-1-2 r \sin \theta+r^{2} \cos 2 \theta\right) \\
& \times \cosh \left(2 r \cos \theta+r^{2} \sin 2 \theta\right) .
\end{aligned}
$$

We also use Maple to verify the correctness of Eq. (29). $>$ evalf(Doubleint $\left(\mathrm{r}^{*} \sin \left(-1-2 \mathrm{r} * \sin (\right.\right.$ theta $)+\mathrm{r}^{\wedge} 2 * \cos (2 *$ theta $)$ $* \cosh \left(2 * r^{*} \cos (\right.$ theta $)+r^{\wedge} 2 * \sin (2 *$ theta $\left.)\right), r=0 . .3$, theta $=0 . .2 *$ Pi));

$$
-23.79203158
$$

$>$ evalf(- $\left.-9 * \mathrm{Pi}^{*} \sin (1)\right)$;

$$
-23.79203158
$$

Also by Eq. (19), we have:

$$
\int_{0}^{2 \pi} \int_{0}^{3} D\left(r, \theta, 1, \frac{\pi}{2}, 2\right) d r d \theta=0
$$

where

$$
\begin{aligned}
D\left(r, \theta, 1, \frac{\pi}{2}, 2\right)= & r \cos \left(-1-2 r \sin \theta+r^{2} \cos 2 \theta\right) \\
& \times \sinh \left(2 r \cos \theta+r^{2} \sin 2 \theta\right) .
\end{aligned}
$$

$>$ evalf(Doubleint $\left(\mathrm{r}^{*} \cos \left(-1-2 \mathrm{r}^{*} \sin (\right.\right.$ theta $)+\mathrm{r}^{\wedge} 2 * \cos (2 *$ theta $\left.)\right)$ $* \sinh \left(2 * r^{*} \cos (\right.$ theta $)+r^{\wedge} 2 * \sin (2 *$ theta $\left.)\right), r=0 . .3$, theta $=0 . .2 *$ Pi), 14);

$$
-1.987124 \cdot 10^{-10}
$$

Example 3 In Eq. (23), let $s=2, \phi=\pi, n=1$, and $R=1$, then:

$$
\int_{0}^{2 \pi} \int_{0}^{1} E(r, \theta, 2, \pi, 1) d r d \theta=\pi \cos (2)
$$

where

$$
E(r, \theta, 2, \pi, 1)=r \cos (-2+r \cos \theta) \cdot \cosh (r \sin \theta) .
$$

$>$ evalf(Doubleint $\left(\mathrm{r}^{*} \cos (-2+\mathrm{r} * \cos (\right.$ theta $)) * \cosh \left(\mathrm{r}^{*} \sin (\right.$ theta ) ), $\mathrm{r}=0 . .1$, theta $=0 . .2 * \mathrm{Pi})$;

$$
-1.307363845
$$

$>\operatorname{evalf}\left(\mathrm{Pi}^{*} \cos (2)\right)$

$$
-1.307363845
$$

On the other hand, using Eq. (24) yields:

$$
\int_{0}^{2 \pi} \int_{0}^{1} F(r, \theta, 2, \pi, 1) d r d \theta=0
$$

where

$$
F(r, \theta, 2, \pi, 1)=r \sin (-2+r \cos \theta) \cdot \sinh (r \sin \theta) .
$$

$>$ evalf(Doubleint $\left(\mathrm{r}^{*} \sin \left(-2+\mathrm{r}^{*} \cos (\right.\right.$ theta $\left.)\right) * \sinh \left(\mathrm{r}^{*} \sin (\right.$ theta )$), \mathrm{r}=0 . .1$, theta $=0 . .2 * \mathrm{Pi})$ );

$$
4.9526896950035 \cdot 10^{-16} \text {. }
$$

\section{Conclusion}

In this paper, we use area mean value theorem to solve some types of double integrals. In fact, the applications of this theorem are extensive, and can be used to easily solve many difficult problems; we endeavor to conduct further studies on related applications. In addition, Maple also plays a vital assistive role in problem-solving. In the future, we will extend the research topic to other calculus and engineering mathematics problems and use Maple to verify our answers.

\section{References}

[1] A. A. Adams, H. Gottliebsen, S. A. Linton, and U. Martin, "Automated theorem proving in support of computer algebra: symbolic definite integration as a case study," Proceedings of the 1999 International Symposium on Symbolic and Algebraic Computation, Canada, pp. 253-260, 1999.

[2] M. A. Nyblom, "On the evaluation of a definite integral involving nested square root functions," Rocky Mountain Journal of Mathematics, Vol. 37, No. 4, pp. 1301-1304, 2007.

[3] C. Oster, "Limit of a definite integral," SIAM Review, Vol. 33, No. 1, pp. 115-116, 1991.

[4] C. -H. Yu, "A study of two types of definite integrals with Maple," Jökull Journal, Vol. 64, No. 2, pp. 543-550, 2014.

[5] C. -H. Yu, "Evaluating two types of definite integrals using Parseval's theorem," Wulfenia Journal, Vol. 21, No. 2, pp. 24-32, 2014.

[6] C. -H. Yu, "Solving some definite integrals using Parseval's theorem," American Journal of Numerical Analysis, Vol. 2, No. 2, pp. 60-64, 2014.

[7] C. -H. Yu, "Some types of integral problems," American Journal of Systems and Software, Vol. 2, No. 1, pp. 22-26, 2014.

[8] C. -H. Yu, "Using Maple to study the double integral problems," Applied and Computational Mathematics, Vol. 2, No. 2, pp. 28-31, 2013.

[9] C. -H. Yu, "A study on double Integrals," International Journal of Research in Information Technology, Vol. 1, Issue. 8, pp. 24-31, 2013.

[10] C. -H. Yu, "Application of Parseval's theorem on evaluating some definite integrals," Turkish Journal of Analysis and Number Theory, Vol. 2, No. 1, pp. 1-5, 2014.

[11] C. -H. Yu, "Evaluation of two types of integrals using Maple, "Universal Journal of Applied Science, Vol. 2, No. 2, pp. 39-46, 2014.

[12] C. -H. Yu, "Studying three types of integrals with Maple, "American Journal of Computing Research Repository, Vol. 2, No. 1, pp. 19-21, 2014

[13] C. - $\mathrm{H}$. Yu, "The application of Parseval's theorem to integral problems," Applied Mathematics and Physics, Vol. 2, No. 1, pp. 49, 2014.

[14] C. -H. Yu, "A study of some integral problems using Maple," Mathematics and Statistics, Vol. 2, No. 1, pp. 1-5, 2014.

[15] C. -H. Yu, "Solving some definite integrals by using Maple, "World Journal of Computer Application and Technology, Vol. 2, No. 3, pp. 61-65, 2014.

[16] C. -H. Yu, "Using Maple to study two types of integrals," International Journal of Research in Computer Applications and Robotics, Vol. 1, Issue. 4, pp. 14-22, 2013.

[17] C. -H. Yu, "Solving some integrals with Maple," International Journal of Research in Aeronautical and Mechanical Engineering, Vol. 1, Issue. 3, pp. 29-35, 2013.

[18] C. -H. Yu, "A study on integral problems by using Maple," International Journal of Advanced Research in Computer Science and Software Engineering, Vol. 3, Issue. 7, pp. 41-46, 2013.

[19] C. -H. Yu, "Evaluating some integrals with Maple," International Journal of Computer Science and Mobile Computing, Vol. 2, Issue. 7, pp. 66-71, 2013.

[20] C. -H. Yu, "Application of Maple on evaluation of definite integrals," Applied Mechanics and Materials, Vols. 479-480 (2014), pp. 823-827, 2013

[21] C. -H. Yu, "Application of Maple on the integral problems," Applied Mechanics and Materials, Vols. 479-480 (2014), pp. 849854, 2013.

[22] C. $-\mathrm{H} . \mathrm{Yu}$, "Using Maple to study the integrals of trigonometric functions," Proceedings of the 6th IEEE/International Conference on Advanced Infocomm Technology, Taiwan, No. 00294, 2013.

[23] C. -H. Yu, "A study of the integrals of trigonometric functions with Maple," Proceedings of the Institute of Industrial Engineers Asian Conference 2013, Taiwan, Springer, Vol. 1, pp. 603-610, 2013. 
[24] C. -H. Yu, "Application of Maple on the integral problem of some type of rational functions," (in Chinese) Proceedings of the Annual Meeting and Academic Conference for Association of IE, Taiwan, D357-D362, 2012.

[25] C. -H. Yu, "Application of Maple on some integral problems, "(in Chinese) Proceedings of the International Conference on Safety \& Security Management and Engineering Technology 2012, Taiwan, pp. 290-294, 2012.

[26] C. -H. Yu, "Application of Maple on some type of integral problem,"(in Chinese) Proceedings of the Ubiquitous-Home Conference 2012, Taiwan, pp.206-210, 2012.

[27] C. - H. Yu, "Application of Maple on evaluating the closed forms of two types of integrals,"(in Chinese) Proceedings of the 17th Mobile Computing Workshop, Taiwan, ID16, 2012.

[28] C. -H. Yu, "Application of Maple: taking two special integral problems as examples,"(in Chinese) Proceedings of the 8th International Conference on Knowledge Community, Taiwan, pp.803-811, 2012.
[29] C. -H. Yu, "Evaluating some types of definite integrals," American Journal of Software Engineering, Vol. 2, Issue. 1, pp. 13-15, 2014.

[30] C. -H. Yu and B. -H. Chen, "Solving some types of integrals using Maple," Universal Journal of Computational Mathematics, Vol. 2 , No. 3, pp. 39-47, 2014.

[31] T. -J. Chen and C. -H. Yu, "A study on the integral problems of trigonometric functions using two methods," Wulfenia Journal, Vol. 21, No. 4, pp. 76-86, 2014.

[32] T. -J. Chen and C. -H. Yu, "Fourier series expansions of some definite integrals," Sylwan Journal, Vol. 158, Issue. 5, pp. 124$131,2014$.

[33] T. -J. Chen and C. -H. Yu, "Evaluating some definite integrals using generalized Cauchy integral formula," Mitteilungen Klosterneuburg, Vol. 64, Issue. 5, pp.52-63, 2014.

[34] R. V. Churchill and J. W. Brown, Complex variables and applications, McGraw-Hill, New York, 1984.

[35] W. R. Derrick, Introductory complex analysis and applications, Academic Press, New York, 1973. 